\title{
CONTENIDO ENERGETICO DE ALGUNOS INVERTEBRADOS BENTONICOS DE LA COSTA DE CHILE Y FLUCTUACION ANUAL EM MYTILUS CHILENSIS HUPE 1854.
}

\author{
WILLIAM E. DUARTE, FERNANDOJARA y CARLOS A. MORENO \\ Institu to de Ecologia y Evolucion. Faćultad de Ciencias. \\ Universidade Austral de Chile, Valdivia, Chile
}

\section{SYNOPSIS}

Caloric values, ash and hydric percentages were obtained from the most abundant benthic invertebrates of Corral Bay, from which this information was not previously available. The range of these values is not different to those of related taxonomic groups of other oceans, Annual fluctuations of these paramenters were studied in Mytilus chilensis so as to obtain a quantitative estimation of the variation of these values.

\section{Introduccion}

En este trabajo se presentan los valores equivalentes entre: el peso seco, peso húmedo y el contenido de energía en gcal obténida en un calorímetro adiabático, de los invertebrados bentonicos más abundantes de Bahía de Corral y del estuario del río Valdivia (Fig. 1). Además en el caso de Mytilus chilensis Hupe se estudia la fluctuación anual de estos parámetros con el objeto de tener una estimación cuantitativa de las variaciones de estos valores.

Estudios similares se han realizado en el hemisferio norte (Tyler, 1973; Wissing et al., 1973) con el mismo tipo de organismos, existiendo la duda si estos valores pueden generalizarse hacia organismos que viven en diferentes condiciones ecológićas en el hemisferio sur. Junto con tratar de despejar esta incógnita se proveen los datos suficientes para emprender estudios de aspectos dinámicos del flujo de energía en estas comunidades bentónicas de la costa de Chile.

\section{Materiales y Metodos}

El material biológico, con excepción de Mytilus chilensis, fué colectado en la Galeta San Carlos, Bahía de Corral (39 $51^{\circ} 38^{\prime \prime}$; $73^{\circ} 26^{\prime} 00^{\prime}$ 'W) (Valdivia, Chile) entre Enero y Junio de 1975 por medio de buceo "SCUBA" a profundidades entre los 2 y 14 metros sobre fondo rocoso. El mismo día de colecta, los animales eran frigorizados $\left(-20^{\circ} \mathrm{C}\right)$ y preservados de esta manera hasta que eran analizados, tiempo que no excedió a los tres meses. Al descongelar los ejemplares se le determinaba el peso húmedo a cada especie; después eran secados a $105^{\circ} \mathrm{C}$ durante 24 horas y secados a peso constante por otras 24 horas en un desecador de $\mathrm{CaSO}_{4}$ (Cummins \& Wuycheck, 1971), después de lo cual se pesaban para obtener de esa manera el peso seco. A continuación, especímenes de la misma especie eran molidos, homogenizados y se comprimían en pas- tillas. Para cada especie se fabricaron 5 pastillas, las que eran pesadas antes de seguir con los siguientes pasos: una era colocada en una mufla hasta combustión completa (4-5 horas) entre $500-510^{\circ} \mathrm{C}$ (Paine, 1964) para obtener el porcentaje de cenizas del peso seco, otra pastilla se colocaba en una estufa a $105^{\circ} \mathrm{C}$ por 24 horas como una manera de corregir el porcentaje de humedad de las otras cuatro pastillas, las otras tres pastillas eran usadas para las determinaciones calorimétricas en un calorímetro adiabático PARR 1241. De cada especie se combustionaron 2 réplicas, o 3, si los valores entre las dos primeras variaban en más de un $1 \%$.

Ao usar la bomba Semi-micro (para muestras muy pequeñas) se combustionaban 3 pastillas si el valor de las 2 primeras difería en más de un $2 \%$. Los valores de las réplicas fueron promediados.

Para el caso de Mytilus chilensis se colectaron muestras mensuales entre Marzo 1975 a Marzo de 1976 provenientes de poblaciones del estuario del río Valdivia.

En este estudio se emplearon tres clases de tamaño, las que fueron fijadas a partir de observaciones de las poblaciones comercializables. Los rangos de tamaño fueron: Clase B: $45.1 \mathrm{~mm}$ a 60.0 $\mathrm{mm}$; Clase C: $60.1 \mathrm{~mm}$ a' $75.0 \mathrm{~mm}$; Clase D: $75.1 \mathrm{~mm}$ y más.

Las conchas fueron limpiadas para eliminar los organismos adheridos, especialmente cirripedios; se extrajo el cuerpo al cual se le determinó posteriormente su peso húmedo. Las muestras fueron colocadas en cápsulas de Petri previamente taradas, puestas en una estufa a $105^{\circ} \mathrm{C}$ por 24 horas, colocadas a continuación en un desecador para enfriar y luego vueltas a pesar. Posteriormente se calculó el porcentaje de humedad de las muestras, siguiendo el procedimento ya indicado. El peso seco fué calculado para cada uno de los ejemplares de cada muestra. Una vez limpias, las conchas fuerom medidas y pesadas. Todos los ejermplares de una misma clase de cada muestra mensual se juntaron, se molieron y se fabricarón pas tillas.

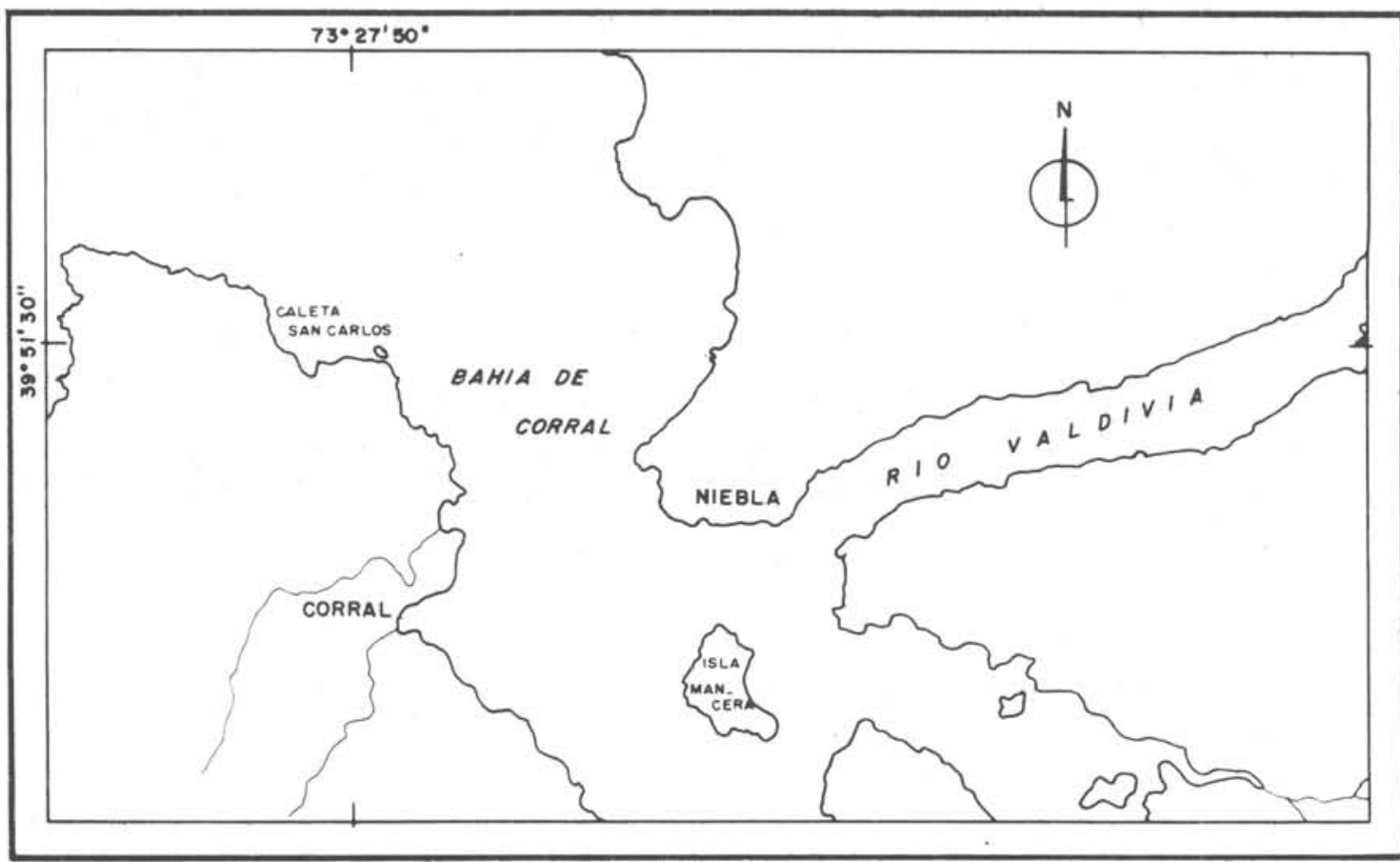

Fig. 1. - Area de muestreo. 
El porcentaje de cenizas de las muestras se obtuvo con idén tico tratamiento al ya mencionado, al igual que lo concemiente á las determinaciones calorimétricas mismas. Se obtuvieron valorés en $\mathrm{gcal} / \mathrm{g}$ de peso seco para cada una de las clases de tamaño a lo largo de un año.

\section{Resultados}

Los valores calóricos obtenidos para las especies analizadas, son consistentes con el rango de variación de los valores previamente conocidos para organismos de grupos taxonómicos afines, los cuales son expresados en calorías por gramo libre de cenizas (Slobod. kin, 1962; Golley, 1961; Tyler, 1973; Wissing et al., 1973). Estos valores pueden cambiar, de acuerdo a su constitucion genética, condición nutritiva e historia de vida (Golley, 1961) y, especialmente en relacion a su madurez sexual (Tyler. 1973). Sin embargo, in fluenciado por el contenido hídrico y materia inorgánica, el contenido energético en peso fresco (peso húmedo) tiende a ser diferente en cada especie (Tab. I).

En el caso del análisis anual desarrollado en Mytlus chilensis se estudiaron los siguientes parámetros:

Peso Seco del Cuerpo

Se estableció un promedio del peso seco en gramos por individuo para cada clase de tamaño durante el período de control

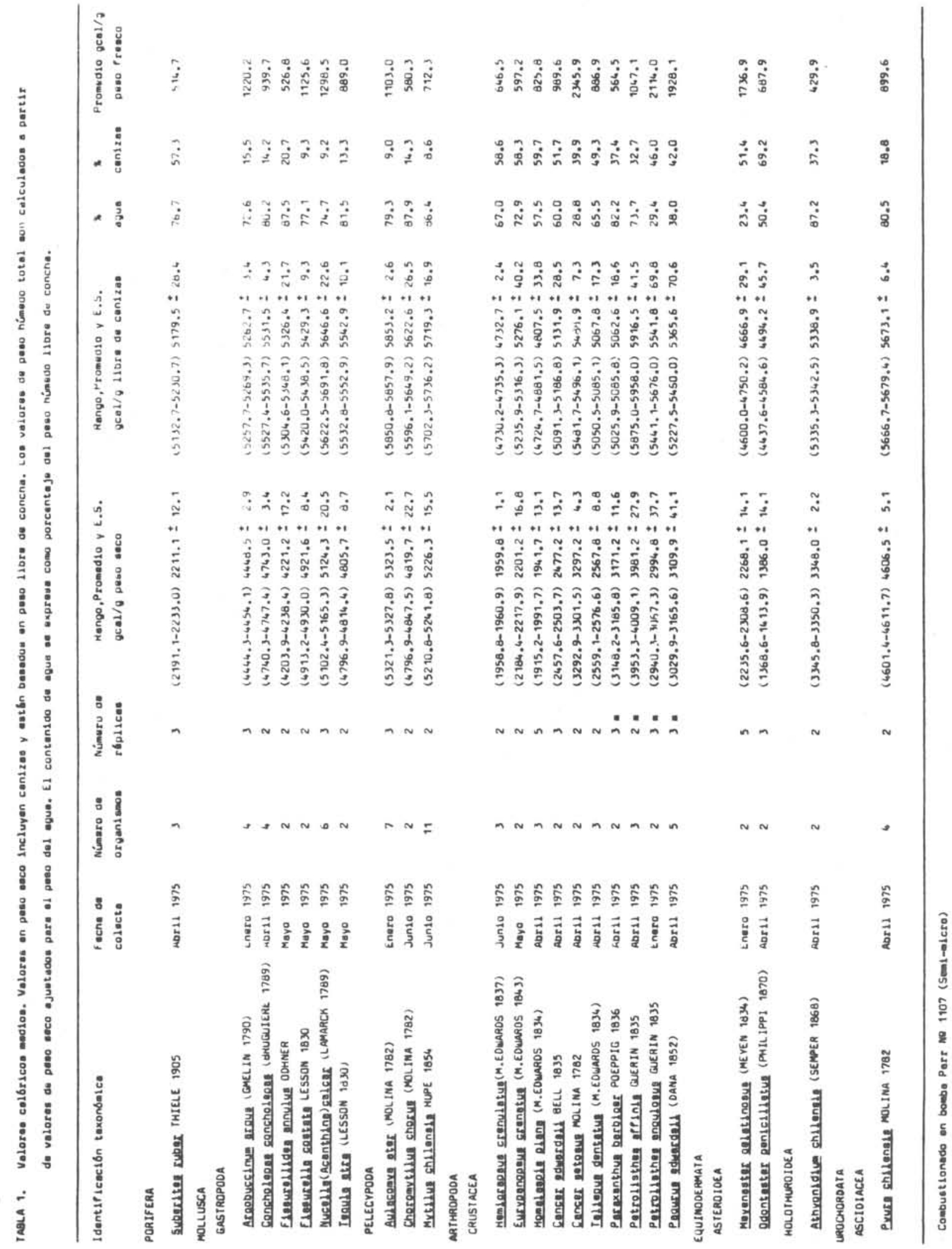


(Fig. 2). Entre los meses de Abril y Julio se observa un descenso paulatino en el peso seco que corresponde al invierno; entre Julio y Octubre hay un aumento gradual, llegando a su máximo valor en el mes de Noviembre, para luego caer bruscamente en Diciembre, cuya representación es la más baja de todo el ciclo anual. En el caso de la Clase B el valor mas bajo es de $0.47 \mathrm{~g}$ en Diciembre. Para la Clase C el valor mas bajo es de $0.83 \mathrm{~g}$ en Diciembre, al igual que para la Clase D que fué de $1.1 \mathrm{lg}$ en el mismo mes.

Los valores de peso seco muestran un pronunciado ciclo anual que relacionamos nor medio del indice de Condición (K)
(Tab. II), que fué calculado empleando la formuia.

$$
K=\frac{W}{1^{3}} \times 10.000 \text {, }
$$

en que W corresponde al peso húmedo del cuerpo del animal y 1 al largo de la concha de cada individuo. El valor calculado en la Tabla II para cada una de las clases de tamano y para cada muestra está expresado como promedio por individuo.

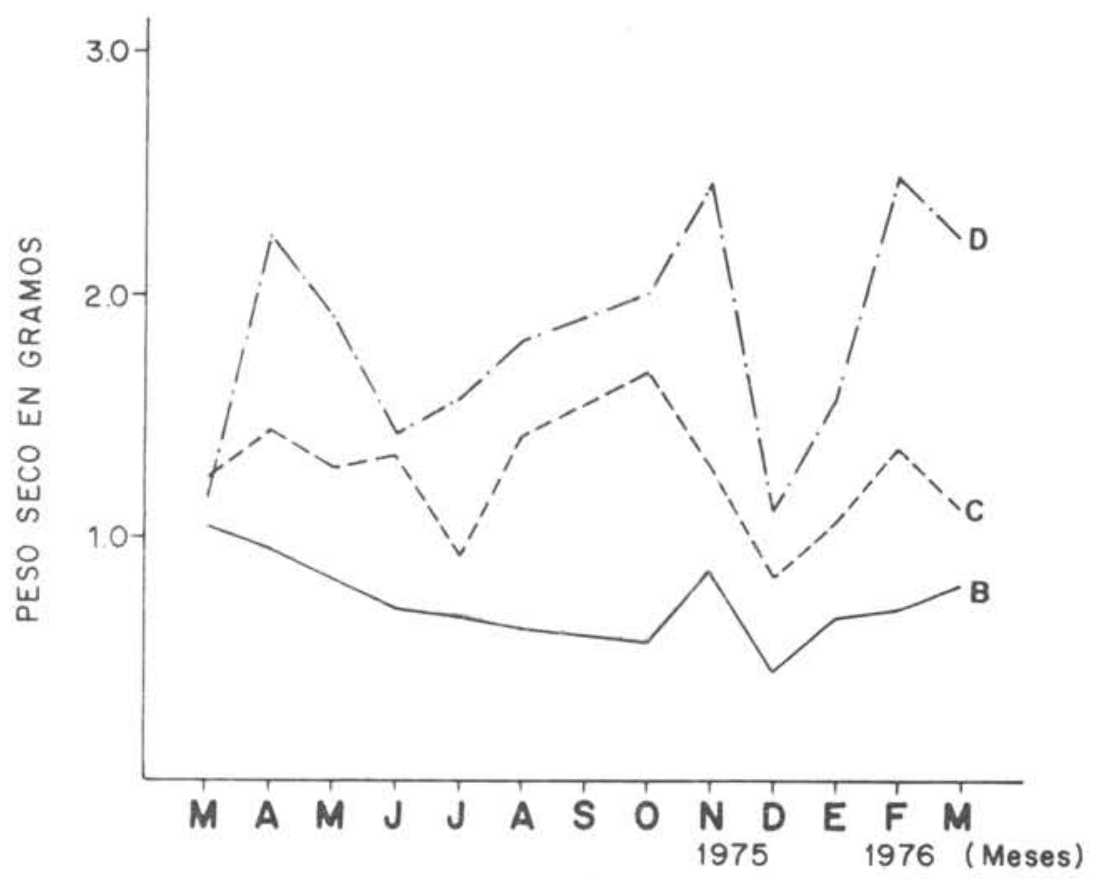

1.1g. 2. - Promedio de peso seco en gramos por individuo para cada clase de tamaño de Mytilus chilensis Hupe del Rio Valdivia.

Tabla 2. Promedio mensual del Indice de Condición ( $\mathrm{K}$ ) de tres clases de tamaño de Mytilus chilensis Hupe del Rio Valdivia.

\begin{tabular}{|c|c|c|c|c|}
\hline & & B & $\mathrm{C}$ & D \\
\hline MARZO & \multirow[t]{10}{*}{1975} & 0.311 & 0.265 & 0.177 \\
\hline ABR1L & & 0.353 & 0.273 & 0.224 \\
\hline MAYO & & 0.311 & 0.291 & 0.263 \\
\hline JUNIO & & 0.268 & 0.259 & 0.210 \\
\hline JULIO & & 0.232 & 0.228 & 0.205 \\
\hline AGOSTO & & 0.273 & 0.278 & 0.229 \\
\hline SEPTIEM & & $\overline{-}$ & - & - \\
\hline OCTUBR & & 0.276 & 0.298 & 0.230 \\
\hline NOVIEM & & 0.314 & 0.273 & 0.272 \\
\hline DICIEMB & & 0.209 & 0.170 & 0.156 \\
\hline ENERO & \multirow[t]{3}{*}{1976} & 0.312 & 0.244 & 0.188 \\
\hline FEBRER & & 0.306 & 0.273 & 0.210 \\
\hline MARZO & & 0.272 & 0.255 & 0.232 \\
\hline
\end{tabular}

\section{Valores Caloricos}

A cadá muestra mensual colectada durante un afo se le hizo determinaciones calorimétricas por clase de tamaño separadamente, obteniéndose ún ciclo de doce meses para cada una. Promedio de calorías por gramo de peso seco por clase de tamaño:

Se analizaron separadamente las muestras en clases de tamaกิ, las que presentan un ciclo anual marcado (Fig. 3)

La tendencia en las tres clases se mantiene semejante a lo largo del aflo registrándose los valores mas bajos en el mes de Febrero, Clase B: $\bar{x} 4735.22 \mathrm{gcal} / \mathrm{g}$ peso seco, Clase C: $\bar{x} 4797.56 \mathrm{gcal} / \mathrm{g}$ peso seco y Clase D: $x 4713.78 \mathrm{gcal} / \mathrm{g}$ peso seco.

Los valores más altos que se obtuvieron para las tres clases corresponden a los de los meses de Octubre, Noviembre y Diciembre, en que el máximo valor es del orden de las $5500 \mathrm{gcal} / \mathrm{g}$ peso seco,

Llama la atención el hecho que precisamente aquellos meses en que el peso promedio por individuo es mas bajo, el valor calórico es el más alto.

Promedio de calorías por gramo de peso seco libre de cenizas por clase de tamaño:
Este análisis corrobora la curva cíclica anual representada en el promedio de calorías por gramo de peso seco para cada una de las clases de tamaño. Si se observa la Figura 4 , puede apreciarse que los valores bajan justamente a la salida del invierno para después llegar a su máxima expresión para las tres clases durante los meses de Octubre a Diciembre, aproximadamente unas $5900 \mathrm{gcal} / \mathrm{g}$ peso seco libre de cenizas, para caer hasta el mes de Febrero aproximadamente a unas $5400 \mathrm{gcal} / \mathrm{g}$ peso seco libre de cenizas, período durante el cual inferimos que estos organismos desovan.

Promedio de calorías por gramo de peso seco libre de cenizas de la población:

La información existente de las tres clases de tamaño separadas se reunió en una sola para ver el comportamiento de las variaciones del promedio de calorías por gramo de peso seco libre de cenizas de la población. Esto está representado en la Figura 5.

\section{Discusion}

Si se observan los diferentes grupos de organismos listados bajo la columna gcal/g peso seco en la Tabla I, llaman la atención 


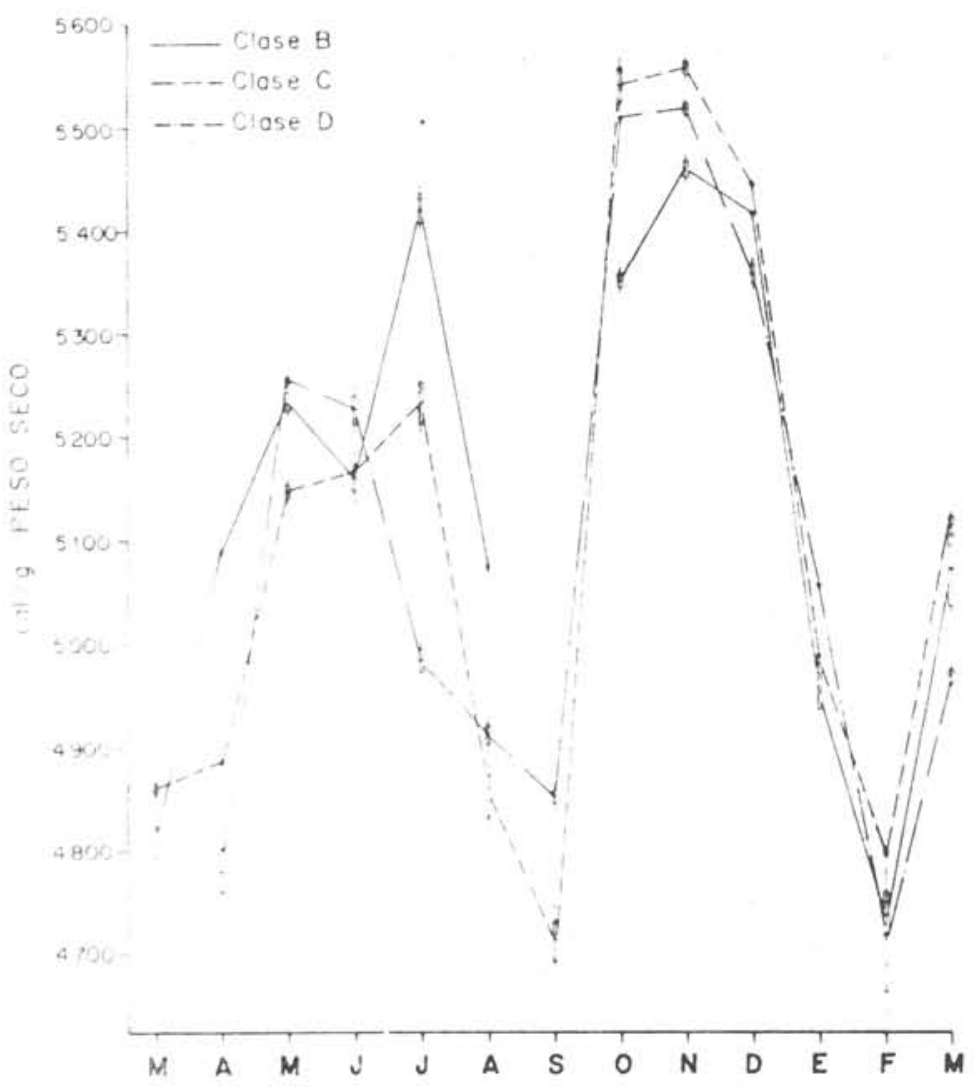

Fig. 3 - Promedio de calorias por gramo de peso seco por clase de tamaño de Mytilus chilensis Hupe del Rio Valdivia.

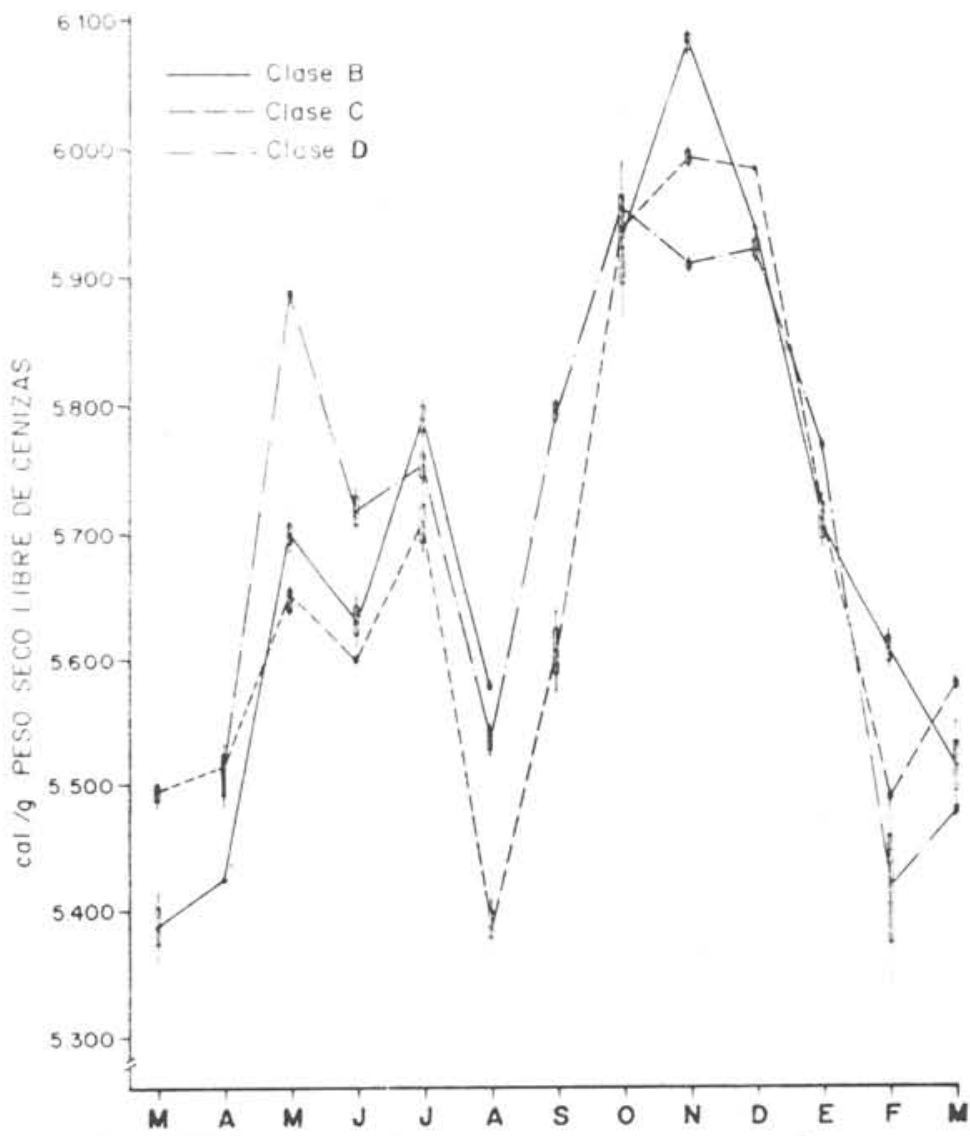

Fig. 4 - Promedio de calorias por gramo de peso seco libre de cenizas por clase de tamafio de Mytilus chilensis Hupe del Río Valdivia. 


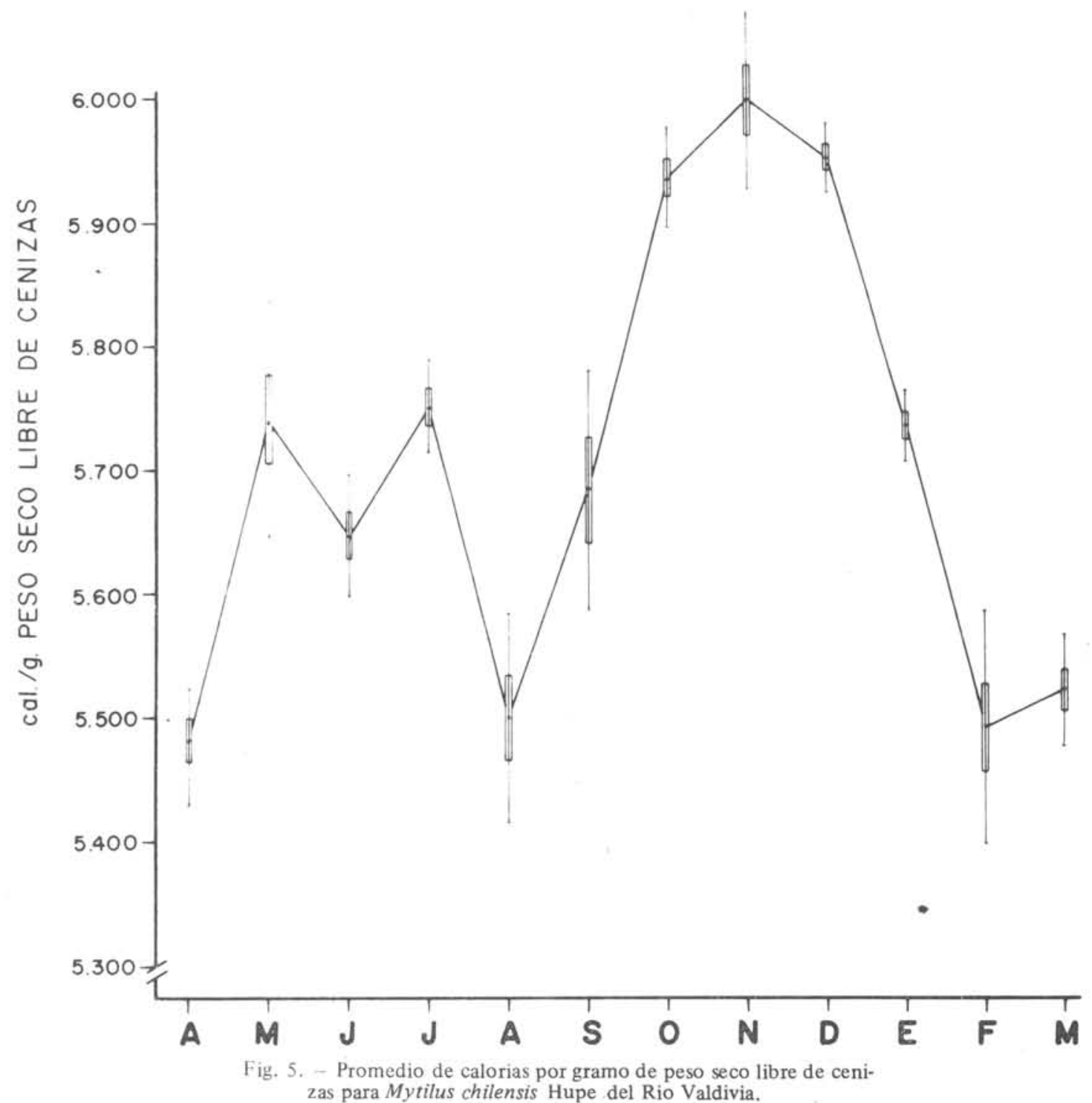

diferencias en los valores promedios. Mollusca (Gastropoda y Pelecypoda), Equinodermata (Holothuroidea) y Urochordata (Ascidiacea) muestran en general, valores mayores que Arthropoda (Crustacea) y Equinodermata (Asteroidea). Por otra parte s se analisa la columna $\mathrm{gcal} / \mathrm{g}$ peso seco libre de cenizas, se ve que los diferentes grupos no varían marcadamente en sus valores promedios. Sin duda que estructuras esqueléticas, especialmente carbonatos presentes en algunos de estos grupos, son responsables de ésto. Una observación directa al porcentaje de cenizas resume y da explicación de lo anterior.

Tyler (1973) llama la atención sobre el hecho de que los valores en $\mathrm{gcal} / \mathrm{g}$ peso seco libre de cenizas tienden a igualar el valor calórico de organismos muy diferentes entre sí, pero que sin embargo la cantidad de materia inorgánica indigerible podría afectar notablemente su valor como alimento para un predador. Justa mente este hecho permite obtener una primera conclusión; si el valor calórico libre de cenizas es similar y los cambios se deben a los elementos inorgánicos que forman cenizas, entonces para estudios de transferencia de energía, basta con estudiar éste factor. Además, si el contenido en cenizas de estos animales es el que hace variar los valores calóricos, cabería preguntarse $\bar{i}$ presenta la materia orgánica variaciones de igual naturaleza?. Al observar el listado en que figuran estos valores (ver $\mathrm{rab} . \mathrm{I}$ ), vemos que hay una homogeneidad notable en los mismos, con valores alrrededor de 5320 calorías.

En el caso de Mytilus chilensis podemos apreciar en la Figura 5 que estos valores para la población presentan una variación anual del orden del $10 \%$, en cambio, las variaciones en el promedio de peso por individuo son del orden del $48 \%$ (Fig. 2). Estas varia ciones en peso por individuo pueden verse reflejadas en los valores del índice de condición (K) en la Tabla II para las tres clases de tamaffo, en que los más bajos aparecen en el mes de Diciembre, fecha en la que estos organismos desovan, lo cual concuerda con Losada (1967) que observó que en la Ensenada de Nercón la liberación de gametos se realiza entre Septiembre y Febrero. Otros meses en que aparecen valores bajos son Junio, Julio y Agosto, época invernal. durante la cual las condiciones del río y del estuario están in fluenciadas por un gran arrastre de sedimentos que provocan un aumento en la turbiedad del agua. Este fenomeno implica una menor luminosidad y por ende una baja en la productividad primaria que incidiría en la robustez de estos filtradores.

Todo esto nos lleva a pensar que el contenido energético de cada gramo del animal presenta una oscilación reducida a lo largo del año $(<10 \%)$. Esta variación se debería también a cambios estacionales en el peso y composición del cuerpo, resultante del almacenamiento $v$ utilización de reservas alimentarias en relación a complejas interacciones de disponibilidad de alimentos y temperatura, con procesos de crecimiento y reproductivos (Dare \& Edwards, 1975).

Em consecuencia, para aplicaciones prácticas de valores calóricos en la estimación de presupuestos y flujo energético en comunidades, sugerimos que no es necesario realizar un análisis calorimétrico anual para todos los organismos, sino más bien, debe darse énfasis a determinaciones puntuales como las presentadas en el listado de la Tabla I.

\section{Agradecimientos}

El financiamiento de la presente contribución ha provenido de la Dirección de Investigación de la Universidad Austral de Chile (Proyecto S-77-34), que los autores agradecen sinceramente. Agradecemos también al PNUMA-Regional America Latina por su ayuda prestada para concurrir al V Simpósio Latinoamericano sobre Oceanografía Biológica en São Paulo, Brasil.

\section{Referencias Bibliográficas}

CUMMINS, K. W. \& WUYCHECK, J. C. 1971. Caloric equivalents 
for investigations in ecological energetics. Mitt. intern. Verein. theor. angew. Limnol., 18:1-15.

DARE, P. J. \& EDWARDS, D. B. 1975. Seasonal changes in flesh weight and biochemical composition of mussels (Mitilus edulis L.) in the Conwy estuary, North Wales. J. exp. mar. Biol. Ecol., 18: 89-97.

GOLLEY, F. B. 1961. Energy values of ecological materials. Eco$\log y, 42(3): 581-584$.

LOSADA, L. E. 1967. Informe sobre madurez sexual y crecimiento de la población de Mytilus edulis chilensis en Mercón, Estero de Castro. (in litteris).
PAINE, R. T. 1964. Ash and calorie determinations of sponge and opisthobranch tissues. Ecology, 45 (2): 384-387.

SLOBODKIN, L. B. 1962. Energy in animal ecology. In: Cragg, J. B., ed. - Advances in ecological research. London, Academic Press, vol. 1, p.69-101.

TYLER, A. V. 1973. Caloric values in some North Atlantic invertebrates. Mar. Biol., 19:258-261.

WISSING, T. E.; DARNELL, R. M.; IBRAHIM, M. A. \& BERNER Jr., L. 1973. Caloric values of marine animals from the Gulf of Mexico. Contr. mar. Sci. Univ. Tex., 17:1-7. 